\title{
Parameter estimation in spatially extended systems: The Karhunen-Lóeve and Galerkin multiple shooting approach*
}

\author{
Anandamohan Ghosh, V. Ravi Kumar ${ }^{\dagger}$, B. D. Kulkarni \\ Chemical Engineering Division, National Chemical Laboratory, Pune 411 008, India
}

(October 31, 2018)

\begin{abstract}
Parameter estimation for spatiotemporal dynamics for coupled map lattices and continuous time domain systems is shown using a combination of multiple shooting, Karhunen-Loéve decomposition and Galerkin's projection methodologies. The resulting advantages in estimating parameters have been studied and discussed for chaotic and turbulent dynamics using small amounts of data from subsystems, availability of only scalar and noisy time series data, effects of space-time parameter variations, and in the presence of multiple time-scales.
\end{abstract}

PACS number(s):05.45.Pq, 05.45.Tp, 05.45.Ra

\section{INTRODUCTION}

In general, extensive systems exhibiting complex spatiotemporal dynamics including chaos may be studied as processes involving reaction-diffusion and convective mechanisms [1]. Analysis of the dynamics of these systems is not an easy task because of the large attractor dimensions involved. It would be desirable to develop ways of studying spatiotemporal systems using reduced model descriptions in conjunction with subsystem dynamics especially when it is known that extensive scaling relationships in dynamics exist as a function of subsystem size [2] 5]. Methods developed for low-dimensional systems may then become applicable with the concomitant advantage of simplifying the data requirements for studying the spatiotemporal dynamics. In this paper, we show a profitable use of this approach for parameter estimation with reduced model descriptions of spatiotemporal systems and which uses subsystem data for the characterization. The low-dimensional models can be obtained by projecting the governing equations onto relevant modes obtained by Karhunen-Loéve (KL) decomposition [6] along with Galerkin projection [7]. Efficient ways such as multiple shooting boundary value algorithms [8 10] that are known to curtail error propagation for low-dimensional chaotic and noisy data for continuous systems [11, may be then reformulated, as shown here, to estimating true parameter values using reduced models for the spatiotemporal dynamics.

There has been a great deal of interest in forecasting spatiotemporal time series and model identification using polynomial and mixed functions 12 14. Short-term prediction of spatiotemporal dynamics by reconstruction of local states [13], and KL decomposition using empirical basis functions by training amplitude coefficients using genetic algorithms for optimization [15] have been studied and assessed. Rather than phase space reconstruction models, identification using some knowledge of the system structure along with nonlinear parametric regression 14 has also been used for modeling spatially extended systems. In this context, the attempt here is to demonstrate the potential of a different approach that uses a multiple shooting algorithm for parameter and state variable estimations in analyzing spatiotemporal behavior.

In Sec. II, the algorithm for spatiotemporal model parameter estimation by a combination of Karhunen-Loéve decomposition, Galerkin's projection and Multiple Shooting (KLGMS) methodology is presented for spatiotemporal systems described by coupled map lattices [16, 17 and partial differential equations. Illustrative examples using the KLGMS approach are presented in Sec. III for a single variable coupled map lattice (CML) that possesses the basic reaction-diffusion and convection mechanisms that give rise to complex patterns including spatiotemporal chaos and convective turbulence. The use of the methodology in characterizing the model from subsystem data using limited number of snapshots is shown and the adaptability of the method in inhomogeneous model [18] identification using perturbation strategies is also discussed. The formalism is then applied in Sec. IV to an autocatalytic reaction-diffusion system described by multivariable partial differential equations (PDE) and exhibiting spatiotemporal chaos [19]. Here we develop the method further for use in stringent situations when only scalar and noisy data in a single variable from subsystems is available for parameter estimation.

${ }^{*}$ This preprint published in Phys. Rev E 64056222 (2001)

${ }^{\dagger}$ e-mail for correspondence: ravi@che.ncl.res.in 


\section{KLGMS APPROACH}

Given snapshots of spatiotemporal data, $u^{(i)}(n, j)$, for variables $i=1,2, \cdots$ at discrete times $(n=1,2, \cdots, M)$ and spatial nodes $(j=1,2, \cdots, L)$, we may obtain the fluctuating components $v^{(i)}(n, j)$ as

$$
v^{(i)}(n, j)=u^{(i)}(n, j)-\bar{u}^{(i)}(j)
$$

where

$$
\bar{u}^{(i)}(j)=\frac{1}{M} \sum_{n=1}^{M} u^{(i)}(n, j)
$$

represent temporal averages for the $M$ number of snapshots considered. The KL decomposition assumes $v^{(i)}(n, j)$ may be expanded in a separable form as

$$
v^{(i)}(n, j)=\sum_{k=1}^{M} a_{k}^{(i)}(n) \phi_{k}^{(i)}(j),
$$

where by truncating the index $k$ to an optimum value, say $N$, it becomes possible to reconstruct the $v^{(i)}(n, j)$ to a required accuracy. In Eq. (3), the $a_{k}^{(i)}(n)$ are time-dependent coefficients while the $\phi_{k}^{(i)}(j)$ are spatial basis functions, satisfying the orthonormality condition

$$
\left(\phi_{l}^{(i)}, \phi_{m}^{(i)}\right)=\delta_{l m}
$$

with the inner product defined as

$$
\left(\phi_{l}^{(i)}, \phi_{m}^{(i)}\right)=\sum_{j=1}^{L} \phi_{l}^{(i)}(j) \phi_{m}^{(i)}(j) .
$$

The spatial basis functions can be empirical basis functions obtained from the spatial correlation matrix [6], Fourier modes, Chebyshev or other sets of orthogonal polynomials, wavelets, etc. [7.20.21]. In particular, to obtain empirical basis functions the KL decomposition of the data $v^{(i)}(n, j)$ [Eq. 3] is carried out in an optimal fashion [7] such that the obtained $\phi_{k}$ are eigenfunctions that maximize the associated eigenvalues $\lambda_{k}=\left\langle\left(\phi_{k}, v\right)^{2}\right\rangle$. Here $(\cdot, \cdot)$ denote the inner product as defined in Eq. (5) while $\langle\cdot\rangle$ represents an averaging procedure that commutes with the inner product [7]. A factor $\eta_{N}=\sum_{l=1}^{N} \lambda_{l} / \sum_{k=1}^{M} \lambda_{k}$ is a measure of the energy content for increasing mode index $k$ and decides an index $N<M$ for which the series in Eq. (3) may be truncated. More often the spatial domain is much too large and estimation of eigenfunctions $\phi_{k}^{(i)}(j)$ from a spatial correlation matrix can become quite involved. The method of snapshots [6] helps to get over this practical difficulty by using instead the temporal correlation matrix $C_{l m}=(1 / M) \sum_{j=1}^{L} v(l, j) v(m, j)$ as the kernel and reduces the KL decomposition to solving a standard eigenvalue problem of the form $C_{l m} \Phi_{m}=\lambda \Phi_{l}$ with $\Phi$ being the required set of eigenfunctions $\left\{\phi_{k}^{(i)}(j)\right\}$. From the $\phi_{k}^{(i)}(j)$ the respective time-dependent coefficients $a_{k}^{(i)}(n)$ may be obtained from

$$
b_{k}^{(i)}(n)=a_{k}^{(i)}(n)=\left(v^{(i)}(n, j), \phi_{k}^{(i)}(j)\right)
$$

and a full description of the dynamics as a series expansion [Eq. (3)] becomes available. Note the introduction of notation $b_{k}^{(i)}(n)$ in Eq. (6) [for $a_{k}^{(i)}(n)$ ] is to convey that the time-dependent coefficients have been obtained solely from transformed snapshot data $v^{(i)}(n, j)$ [Eq. (11)]. The known values of $b_{k}^{(i)}(n)$ may, therefore, be used as time-series for model characterization and parameter estimation studies.

Our next step is to obtain a simpler model of the spatiotemporal system that can be used for the purposes of parameter estimation. Such a reduced description may be derived by Galerkin's projection of the model in conjunction with the KL expansion [Eq. (3)] and we shall discuss the steps involved in this procedure separately for discrete CML type systems and for continuous systems modeled by PDEs.

A CML model with discrete space index $j$ and time $n$ may be written in a general form as

$$
u^{(i)}(n+1, j)=g\left(u^{(1)}\left(n, j+j^{\prime}\right), u^{(2)}\left(n, j+j^{\prime}\right), \cdots, \mu\right)
$$


where, $j^{\prime}=\cdots,-2,-1,0,1,2, \cdots$ and $g$ any function with $\mu=\left\{\mu_{1}, \mu_{2}, \cdots, \mu_{p}\right\}$ the model parameters. The boundary conditions for Eq. (7) are generally dependent on the example being studied and in Sec. III we show cases involving periodic and open flow (as in convection) boundary conditions. In the KL-Galerkin's method we minimize the residual by forcing the projection of the model on the subspace of truncated basis functions $\phi_{k}^{(i)}(j)$ to be zero at all time $n$ and obtain $N<M$ KL-Galerkin equations for the CML model described by Eq. (7) as

$$
a_{k}^{(i)}(n+1)=\sum_{j=1}^{L}\left[g\left(\sum_{l=1}^{N} a_{l}^{(1)}(n) \phi_{l}^{(1)}(j)+\bar{u}^{(1)}(j), \cdots, \mu\right)-\bar{u}^{(i)}(j)\right] \phi_{k}^{(i)}(j)
$$

where the use of the orthonormal property of the basis functions [Eq. (11)] allows considerable simplification. The initial conditions for solving the map [Eq. (8)] can be obtained by forcing the initial residual to have zero projection on the space of basis functions, i.e., $a_{k}^{(i)}(0)=\sum_{j=1}^{L} v^{(i)}(0, j) \phi_{k}^{(i)}(j)$.

For continuous systems in both space $x$ and time $t$, the mathematical model can be written in general form as PDE's with appropriate boundary conditions, viz.,

$$
\dot{u}^{(i)}(t, x)=h\left(u^{(1)}(t, x), u^{(2)}(t, x), \cdots, \mu\right)
$$

and the corresponding KL-Galerkin equations by projection obtained as

$$
\dot{a}_{k}^{(i)}(t)=\int h\left(\sum_{l=1}^{N} a_{l}^{(1)}(t) \phi_{l}^{(1)}(x)+\bar{u}^{(1)}(x), \cdots, \mu\right) \phi_{k}^{(i)}(x) d x .
$$

Here again $N<M$ equations form a simpler and reduced model incorporating system parameters $\mu$. Note that the summation in Eq. (5) for discrete systems becomes an integral for continuous ones, i.e., $\left(\phi_{l}^{(i)}, \phi_{m}^{(i)}\right)=$ $\int_{0}^{1} \phi_{l}^{(i)}(x) \phi_{m}^{(i)}(x) d x$ with the $(\cdot, \cdot)$ now denoting the usual $\mathbf{L}^{2}([0,1])$ inner product space [7] defined in spatial domain $0 \leq x \leq 1$. The initial conditions for solving Eq. (10) may again be independently obtained by $a_{l}^{(i)}(0)=$ $\int v^{(i)}(0, x) \phi_{l}^{(i)}(x) d x$.

We next discuss the use of the simpler KL-Galerkin models in the time-dependent coefficients $a_{k}^{(i)}(n)$ [i.e., [Eq. (8) for CML or Eq. (10) for a continuous system], along with the known coefficient values $b_{k}^{(i)}(n)$ obtained from the data by [Eq. (6)] for the estimation of parameters $\mu$ for the respective spatiotemporal dynamics [i.e., Eq. (7) or Eq. (9)]. We show this is possible by using an effective algorithm in parameter estimation for low-dimensional nonlinear dynamical systems, viz., the multiple shooting algorithm formulated as a multipoint boundary value problem with nonlinear constraints for optimization [8,11]. The algorithm curtails error propagation observed in chaotic dynamics and offers advantages in terms of number of data points required, negating effects of noise, handling missing data situations, parallelization and stopping at local minima during optimization [9, 10].

For the CML model [Eq. (7)], the observations in the discrete time interval $\left[n_{1}, n_{M}\right]$ may be chosen to form a grid for $M$ multiple shooting points at $n_{1}<n_{2}<\cdots<n_{l}<\cdots<n_{M}$ modes forming $(M-1)$ sets of initial value problems in the form of [Eq. (8)] for each of the shooting nodes $n_{l}$ with $1 \leq l \leq M-1$. That is, on considering $k=1,2, \cdots, N$ spatial basis modes for $N<M$, we obtain for a $i^{\text {th }}$ variable $N(M-1)$ maps to be solved

$$
a_{k}^{(i)}(n+1)=\mathcal{G}\left(a_{k}^{(i)}(n), \phi_{k}^{(i)}(j), \bar{u}^{(i)}(j), \mu\right) \quad a_{k}^{(i)}\left(n_{l}\right)=s_{k}^{(i)}(l),
$$

for an incremental time-step $n \rightarrow n+1$. Here, $s_{k}^{(i)}(l)$ denotes the value of the $i^{\text {th }}$ variable at the $l^{\text {th }}$ shooting-point for $k^{\text {th }}$ basis mode and initial guesses for solving $N(M-1)$ maps of Eq. (11) are taken to be the known values of $b_{k}^{(i)}(l)$ [Eq. (包)]. It may be noted that for the system governed by PDEs and data available at shooting points $\tau_{1}<\tau_{2} \cdots<\tau_{l}<\cdots<\tau_{M}$ and that are monitored at time $n \Delta t$, the corresponding set of $N(M-1)$ initial value problems may be written as

$$
\dot{a}_{k}^{(i)}(t)=\mathcal{H}\left(a_{k}^{(i)}(t), \phi_{k}^{(i)}(x), \bar{u}^{(i)}(x), \mu\right), \quad a_{k}^{(i)}\left(\tau_{l}\right)=s_{k}^{(i)}(l) .
$$

We can construct an augmented vector of initial values $s$ and parameters $\mu$ for either model Eq. (『) or Eq. (9), viz.,

$$
\mathbf{z}=\left(s_{1}^{(1)}(1), s_{1}^{(1)}(2), \cdots, s_{1}^{(1)}(M), s_{1}^{(2)}(1), s_{1}^{(2)}(2), \cdots, \mu_{1}, \mu_{2}, \cdots, \mu_{p}\right)
$$

and attempt to minimize a least square cost function $\mathcal{L}_{2}(\mathbf{z})$ of the form 


$$
\mathcal{L}_{2}(\mathbf{z})=\sum_{i=1,2, \cdots} \sum_{k=1}^{N} \sum_{n=1}^{M} \frac{1}{\sigma_{k n}^{(i)}}\left[b_{k}^{(i)}(n)-\mathcal{F}_{k}^{(i)}(a(n), \mu)\right]^{2},
$$

where, $\mathcal{F}_{k}^{(i)}$ is a function relating components of $\mathcal{G}$ in Eq. (11) or $\mathcal{H}$ in Eq. (12) and comparing to the known $b_{k}^{(i)}(n)$ with $\sigma_{k n}^{(i)}$ the square of the standard deviation. The minimization in Eq. (14) is carried out subject to satisfying

$$
a^{(i)}\left(n_{l+1}\right)-s^{(i)}(l+1) \rightarrow 0
$$

so that the trajectories in the coefficients $a^{(i)}\left(n_{l+1}\right)$ become continuous. Alternatively stated, by identifying $\mathbf{y}_{1}(\mathbf{z})=r\left(s_{1}^{(i)}(1), s_{2}^{(i)}(2), \cdots, s_{N}^{(i)}(M), \mu_{1}, \mu_{2}, \cdots, \mu_{p}\right)$ and $\mathbf{y}_{2}(\mathbf{z})=a^{(i)}\left(n_{l+1}\right)-s^{(i)}(l+1)$ we obtain, a standard nonlinear minimization problem [22] of the type

$$
\min _{\mathbf{z}}\left\{\left\|\mathbf{y}_{1}(\mathbf{z})\right\|_{2}^{2} \mid \mathbf{y}_{2}(\mathbf{z}) \rightarrow 0\right\}
$$

where the minimization of $\mathbf{y}_{1}(\mathbf{z})$ corresponds to minimizing the cost function Eq. (14) while that for $\mathbf{y}_{2}(\mathbf{z})$ implies satisfying the constraints imposed by Eq. (15). The minimization of Eq. (16) can be carried out by starting with initial guess values $\mathbf{z}^{(0)}$ and iterating for $\mathbf{z}$ using $\mathbf{z}^{(q+1)}=\mathbf{z}^{(q)}+\omega^{(q)} \Delta \mathbf{z}^{(q)}$ where, $\omega^{(q)} \in[0,1]$ are damping factors. In doing so corrections to the augmented vector $\mathbf{z}$, viz., $\Delta \mathbf{z}^{(q)}$ are obtained by solving the linearized problem

$$
\min _{\mathbf{z}}\left\{\left\|\mathbf{y}_{1}\left(\mathbf{z}^{(q)}\right)+\frac{\partial \mathbf{y}_{1}\left(\mathbf{z}^{(q)}\right)}{\partial \mathbf{z}} \Delta \mathbf{z}^{(q)}\right\|_{2}^{2} \quad \mid \mathbf{y}_{2}\left(\mathbf{z}^{(q)}\right)+\frac{\partial \mathbf{y}_{2}\left(\mathbf{z}^{(q)}\right)}{\partial \mathbf{z}} \Delta \mathbf{z}^{(q)} \rightarrow 0\right\}
$$

The above Eq. (17) may be solved by a suitable nonlinear optimization technique in the optimization variables, $\mathbf{z}$ [Eq. (13] for arbitrary guess values for the parameters $\mu$ and initial states $s_{k}^{(i)}(l)=b_{k}^{(i)}(l)$. In the coding of the above KLGMS approach, we have employed the successive quadratic programming algorithm 23] coupled with numerical differentiation for the sensitivity matrices. For illustration, we have retained simplicity in the cost function [Eq. (14)] but more effective functionals [24] may be adopted in the optimizing for the parameters $\mu$. It is to be noted that the methodology also allows optimizing for the $s_{k}^{(i)}(l)$ even when some values of $b_{k}^{(i)}(l)$ are initially not available and arising due to missing snapshot data in say some $i^{\text {th }}$ variable. The illustrative examples presented in Sec. III and Sec. IV bring out further the advantages offered by KLGMS approach when snapshot data availability is limited and possibly noise contaminated.

\section{PARAMETER ESTIMATION USING KLGMS FOR CML}

Because of their computational simplicity, CMLs are a popular and convenient paradigm for studying fully developed turbulence [16, 17, 25], chaos [26], and pattern formation [1] in systems. A CML model is a discrete space-time system with continuous state space and studies the effects of local nonlinear reaction dynamics, the coupling arising from diffusion due to state space gradients as well as convective effects by asymmetric coupling [17]. Here, we consider a CML involving a single spatial dimension and incorporating these mechanisms as

$$
\begin{array}{r}
u(n+1, j)=\left(1-D_{d}-D_{c}\right) f(u(n, j))+D_{c} f(u(n, j-1)) \\
+\frac{D_{d}}{2}[f(u(n, j+1))+f(u(n, j-1))],
\end{array}
$$

where, $u(n, j), j=1,2, \cdots, L$ is the state of the variable located at site $j$ at time $n$ for a lattice of size $L, D_{d}$ the nearest neighbor diffusive coupling strength, and $D_{c}$ denoting the asymmetric coupling constant. This being a single variable system we suppress the index $(i)$ in this Section. For $D_{c}=0$ the system represents a reaction-diffusion system while for $D_{c} \neq 0$ mimics one with convective effects included. We assume the reaction dynamics on the lattice sites is governed by the nonlinear logistic function $f(u)=1-F u^{2}$, where, $F$ is the nonlinearity parameter. Thus, depending on the parameter values for $F, D_{d}$ and $D_{c}$, a variety of dynamical patterns may be observed in Eq. (18) and characterized as in [17]. We bring out the methodology for estimating parameters for selected dynamics covering a broad range of complexity, viz., (a) weak chaos; (b) traveling wave; (c) fully developed chaos; and (d) convective turbulence. Spatiotemporal data for the different cases are obtained by evolving Eq. (18). All the sites are given random initial conditions at $n=0$ and snapshots are stored after eliminating initial transients. Cases $(a, b, c)$ are evolved with periodic boundary conditions, i.e., $u(n, 1)=u(n, L)$ while for the convective case $(d)$ the left boundary is assumed fixed, i.e., $u(n, 1)=1$, with the right boundary open. The gray-scale images of the spatiotemporal data 
with the parameter values yielding the data for a lattice size of $L=60$ and for $M=20$ snapshots, is shown in Fig. 1. In studies involving subsystems, only the data corresponding to the evolution of the chosen subsystems are stored.

We obtain a KL decomposition for the spatiotemporal data $v(n, j)$ and Table 1 i shows the corresponding eigenvalues $\lambda_{k}$, and the energy content $\eta_{k}$, for the data shown in Fig. 1 $(a-d)$. The results show that for the CML exhibiting weak chaos and travelling wave, a smaller number of basis modes $N=3$ and $N=5$, respectively, are required to capture and reconstruct $99 \%$ of the data. For the more complex patterns, viz., fully developed chaos and convective turbulence the number of basis modes significantly rise to 15 for $\approx 99 \%$ and 19 for $\approx 100 \%$ accuracy.

Studies with KL-Galerkin Eq. (8) for the CML Eq. (18) using the KLGMS approach did accurately and simultaneously estimate the unknown parameters $\left(F, D_{d}, D_{c}\right)$ from a few snapshots of the data. The results of convergence for arbitrary and different initial guesses for the parameters shown in Table II for the (a) weakly chaotic, $(b)$ travelling wave and (c) fully developed chaos cases. The robustness is seen when parameters were successfully estimated even for noisy spatiotemporal data sets [Table II] obtained by additive noise $\hat{u}(n, j)=u(n, j)+\eta$ with Gaussian distribution noise $\eta \in N\left[0, \varepsilon^{2}\right]$. The strength of the noise level used was determined by $\sigma_{\text {noise }} / \sigma_{\text {data }}$ and chosen to be 0.01. It may be observed that noise in the data enters through the "coefficient trajectories" $b_{k}(l)$ that are obtained by the convolutions of the fluctuating data $v(n, j)=u(n, j)-\bar{u}(j)$ with the basis functions $\phi_{k}^{(i)}(j)$ via, Eq. (6). Note that although the functional form of the CML in the form of Eq. (18) is single dimensional and single variable the procedure may be extended to situations involving multivariable mappings Eq. (7) and higher spatial dimensions. The effects of considering higher spatial dimensions do not change the methodology because the KL expansion yields two or three-dimensional spatial basis functions $\phi(i, j, k)$ but the Galerkin equation still retains the mapping form of Eq. (8) in the time-dependent coefficients $a_{k}(n)$. Applications of the method to systems with multivariable coupling and scalar data are shown in the Sec. IV studying KLGMS for continuous time systems. For brevity the results obtained with CMLs on these aspects are not presented.

The presence of scaling relationships in Lyapunov exponents as a function of subsystem size have been studied [3 5 . For KL decomposition modes, using the spatial correlation matrix, a linear relationship in KL dimension [27] has also been seen. Our studies for subsystem scaling with the temporal correlation matrix, $C_{l m}$, showed some interesting features. We observe that $\mathcal{D}_{T}=\max \left\{N: \eta_{N} \leq f\right\}$ required to capture a fraction $f$ of the total variance showed scaling behavior after an optimum subsystem size before saturation. The saturation occurs either due to the dynamics being not complicated enough to warrant all modes to be included as a function of subsystem size or alternatively when the dynamics is sufficiently complex that all KL modes (limited by the number of snapshots $M$ ) are required. Therefore, depending on the complexity of the pattern and number of snapshots, $M$, an optimum subsystem size exists beyond that only system features can be extracted reliably. The feasibility of estimating parameters by relaxing the need for data from the entire spatial domain was then considered. Thus, on computing $\mathcal{D}_{T}$ for the convective CML data [Fig. $[(d)$ ] as a function of subsystem size $j$ for $L=80$ we observed that beyond $j=30$ there is linear scaling and this determines the optimum subsystem size. For this subsystem size even with a lower number of modes $(\approx N=15)$, parameter values could be estimated, while for larger subsystem size all KL modes need to be considered. Figure 2(a) shows the subsystem data for the central 31 lattice sites and used for parameter estimation purposes for $N=15$. The accurate convergence of the estimated parameters $F, D_{d}$ and $D_{c}$ with search iterations is shown in Fig. 2( $(b-d)$ and reported as the homogeneous case (a) in Table III. These studies suggest that when reliability of data is poor from certain regions, considerable information may be gained by using only authentic data available from other subsystems in the spatial domain.

A number of real situations have inhomogeneous distribution of parameter values in space and/or slowly varying in time domain. Studies in this context for parameter estimation were carried out and the analysis of a simple example is discussed here. We evolve a CML such that the sites in the left half (i.e., $1 \leq j \leq 256$ ) have $F=2.0$ while the right half (i.e., $257 \leq j \leq 512)$ evolve data with $F=1.9$ for $L=512$. Subsystem data from each half [Fig. 3] was used for parameter estimation. Since the local dynamics propagate in space, the data obtained from both subsystems had composite features leading to inconsistent and unreliable parameter estimates. To overcome this difficulty we recorded data immediately after a giving a perturbation at time $n$ (i.e., noise of strength 0.01) to the variable $u(n, j)$ and then carried out KLGMS parameter estimation for each of the subsystems (left and right). The results presented in Table III cases $b, c$ show that parameter estimation is now possible. Studies were also carried out for situations modeling $F$ as a slowly varying parameter in time. The need to record subsystem data at optimum time gaps was found necessary to monitor the slow parametric changes. In real situations, repeated parameter estimations at sufficient time intervals can help in establishing relationships in the nature of parametric variations and this can considerably aid system analysis. 


\section{PARAMETER ESTIMATION USING KLGMS FOR REACTION-DIFFUSION SYSTEM}

A basic problem in studying spatially extended dynamical systems is the quantitative comparison of experimental data with models based on partial differential equations. For example, in the study of pattern forming systems, the theoretical models usually take the form of reaction-diffusion equations that have been studied both theoretically and experimentally [1,28,29]. For our study of parameter estimation we shall illustrate the methodology for a prototype reaction-diffusion model where one chemical species grows autocatalytically on another species [30,19]. This model is a simplification of the model of glycolysis proposed by Selkov [31] and it follows the reaction mechanism $U+2 \mathrm{~V} \rightarrow 3 \mathrm{~V}$; $V \rightarrow P$ with a continuous supply of the reactant $U$ and removal of product $P$. The model has been extensively studied from the point-of-view of pattern formation and comparisons with features observed in experimental data have also been attempted [32].

The reaction-diffusion mechanism yields a two variable PDE model involving concentrations $u^{(1)}(t, x), u^{(2)}(t, x)$ of $U, V$, respectively, and for a spatially one dimensional system, we obtain:

$$
\begin{aligned}
& \frac{\partial u^{(1)}(t, x)}{\partial t}=D_{u} \nabla^{2} u^{(1)}(t, x)-u^{(1)}(t, x)\left(u^{(2)}(t, x)\right)^{2}+f\left[1-u^{(1)}(t, x)\right] \\
& \frac{\partial u^{(2)}(t, x)}{\partial t}=D_{v} \nabla^{2} u^{(2)}(t, x)+u^{(1)}(t, x)\left(u^{(2)}(t, x)\right)^{2}-[f+k] u^{(2)}(t, x) .
\end{aligned}
$$

Here, $D_{u}$ and $D_{v}$ are the diffusion coefficients of species $U$ and $V$, with parameters $f$ and $k$ related to the flow of reactant into the system and the kinetic rate constant. The parameters $f, k$ form a pair of bifurcation parameters that may be varied to obtain a host of spatiotemporal Turing patterns for unequal diffusion coefficients of the chemical species as seen in 19 .

In our study, we consider the situation corresponding to system exhibiting spatiotemporal chaos Fig. 1. as studied in [19]. For obtaining the spatiotemporal data $u^{(1)}(t, x), u^{(2)}(t, x)$, Eq. (19) is solved numerically with Euler discretization in the spatial domain, with spatial length $L=1$ spanning 160 spatial sites and $M=40$ snapshots are stored at a time step $\Delta t=0.1$ and with periodic boundary conditions $u^{(1)}(t, 0)=u^{(1)}(t, L)$ and $u^{(2)}(t, 0)=u^{(2)}(t, L)$ imposed. The initial conditions correspond to the stationary solution $u^{(1)}(0, x)=1$ and $u^{(2)}(0, x)=0$ except for a few central sites that are given a random perturbation to break the symmetry.

Here we will also consider situations where only scalar data in a single variable $u^{(1)}(t, x)$ is monitored. Because the data in the $u^{(2)}(t, x)$ is not available we need to use basis functions other than empirical. In the present study, we choose to exemplify KLGMS using Fourier basis functions defined as

$$
\phi_{k}^{(i)}(x)=\sqrt{2} \sin (2 \pi k x)
$$

with temporal coefficients obtained by

$$
b_{k}^{(i)}(t)=a_{k}^{(i)}(t)=\int_{0}^{L} v^{(i)}(t, x) \phi_{k}^{(i)}(x) d x
$$

and use the $b_{k}^{(i)}(t)$ as observables in evaluating the least square functional in Eq.(14). For the model Eq. (19) the KL Galerkin projection equations for the time-dependent coefficients, i.e., Eq. (10), for modes $k=1,2, \cdots, N$ can be written as (suppressing $(x)$ and $(t)$ ):

$$
\begin{array}{r}
\dot{a}_{i}^{(1)}=\int_{0}^{L}\left[D_{u} \nabla^{2}\left(\sum_{k=1}^{N} a_{k}^{(1)} \phi_{k}^{(1)}+\bar{u}^{(1)}\right)-\left(\sum_{k=1}^{N} a_{k}^{(1)} \phi_{k}^{(1)}+\bar{u}^{(1)}\right)\left(\sum_{k=1}^{N} a_{k}^{(2)} \phi_{k}^{(2)}+\bar{u}^{(2)}\right)^{2}\right. \\
\left.+f\left(1-\sum_{k=1}^{N} a_{k}^{(1)} \phi_{k}^{(1)}-\bar{u}^{(1)}\right)\right] \phi_{i}^{(1)} d x \\
\dot{a}_{i}^{(2)}=\int_{0}^{L}\left[D_{v} \nabla^{2}\left(\sum_{k=1}^{N} a_{k}^{(2)} \phi_{k}^{(2)}+\bar{u}^{(2)}\right)+\left(\sum_{k=1}^{N} a_{k}^{(1)} \phi_{k}^{(1)}+\bar{u}^{(1)}\right)\left(\sum_{k=1}^{N} a_{k}^{(2)} \phi_{k}^{(2)}+\bar{u}^{(2)}\right)^{2}\right. \\
\left.-(f+k)\left(\sum_{k=1}^{N} a_{k}^{(2)} \phi_{k}^{(2)}+\bar{u}^{(2)}\right)\right] \phi_{i}^{(2)} d x
\end{array}
$$


and a reduced $N<M$ set of ODEs solved by integrating using the initial conditions discussed for Eq. (10).

Our studies with the set of Galerkin equations Eqs. (22) with KLGMS for estimating system parameters using the spatiotemporally chaotic data (Fig. (4) showed two interesting features described below. First, accurate parameter estimation of the diffusion coefficients $\left(D_{u}, D_{v}\right)$ did not particularly depend on the choice of $(k, f)$ when initial transient data were chosen as snapshots with the diffusion mechanism playing a significant role. It was also observed that similar results in $(k, f)$ were obtained using snapshots after giving a perturbation to the system state $u^{(1)}(t, x)$ at any time $t$. The second feature was that having evaluated $\left(D_{u}, D_{v}\right)$ in the above fashion the other two parameters $(f, k)$ could be successfully estimated using post-transient data. These observations suggest that diffusion rates and reaction rates occur at differing time-scales and clearly point to the need for suitable data sampling strategies. It may be noted that the values of diffusion coefficients employed here lie in typical ranges. The multiple time-scale features discussed above may therefore be expected to be frequently present in the dynamics of spatiotemporal systems. Any methodology seeking model identification would need to consider this relevant aspect for parameter estimation.

Without any ambiguity, we discuss other features of the KLGMS with reference to evaluating $f$ and $k$ from monitored post-transient data. The KL decomposition of the data set using Fourier modes for 40 snapshots showed that a single Fourier basis mode could reconstruct the data snapshots accurately $(>99.8 \%)$. The results of parameter estimation with this single mode considered showed that accurate convergence was consistently possible even when the data was corrupted with noise of the order of $5 \%$ and are summarized in Table IV case $a$. For the present reaction-diffusion system we have observed that the use of basis functions with the known Fourier form allows tolerance for higher noise levels when compared to empirical basis functions (using correlation matrices). A more practical problem arises in multivariable systems when only one dynamical variable is monitored. We assume that $u^{(2)}(t, x)$ is not monitored and assign initial guesses for the temporal coefficients $a^{(2)}(t)=0.2$ and $\bar{u}^{(2)}=0$ for the multiple shooting algorithm. The least square functional Eq. (14) and equality constraints are suitably modified so as to take into account only terms in variables $u^{(1)}(t, x)$. Results of the study presented in Table IV case $b$ showing accurate parameter estimation is again possible for both $f, k$ although with a small decrease in noise tolerance. It may be seen that the parameter estimation of $k$ present only in the $u^{(2)}$ equation of the PDE model Eq. (19) is also possible. Importantly, we have recovered the unmonitored variable $u^{(2)}(t, x)$ using Eq. (3) and estimated the values of $a^{(2)}(t)$ by multiple shooting.

Similar to the studies using CML we attempted to evaluate parameters using subsystem data with only scalar variable data in $u^{(2)}(t, x)$ available. An indication of the optimum subsystem size in this study using Fourier basis functions was suggested on evaluating the normalized power $P\left(n_{s}\right)=\int\left[a_{1}^{(1)}(t)\right]_{n_{s}}^{2} d t$ as a function of the subsystem size $n_{s}$ and is shown in Fig. 周. The results indicate a near saturation beyond $n_{s}=80$. The results of KLGMS carried out with subsystem scalar data available only in $u^{(1)}$ and with noise (Table IV case $c$ ) shows that parameter estimation within reasonable error bounds is still possible.

\section{CONCLUSION}

The results obtained using the KLGMS show that this basic framework has the necessary robustness for parameter estimation for spatiotemporal dynamics. We exemplify the methodology by simultaneously estimating all parameters of a CML and a reaction-diffusion system. Importantly, for complex dynamics and noise in the data we show that accurate parameter estimates are possible even from small data samples obtained from subsystems of optimal size. We show ways of adapting the methodology for inhomogeneous situations when parameters vary in space and time and by using transient data soon after perturbing the system dynamics. The usefulness of this strategy especially when multiple time-scales are present in the system dynamics has been discussed. The algorithm can be extended to situations when only scalar data is available and has the capability to recover the dynamics of the unmonitored variable. The study presented here should also help in the analysis and design of experiments for spatiotemporal systems that are often costly and difficult to perform.

\section{ACKNOWLEDGMENTS}

Authors wish to thank Unilever Research, Port Sunlight, UK, for financial assistance in carrying out the work. 
[1] M. C. Cross and P. C. Hohenberg, Rev. Mod. Phys. 65, 851 (1993).

[2] P. Grassberger, Phys. Scr. 40, 336 (1989).

[3] M. Bauer and W. Martienssen, J. Phys. A 24, 4557 (1991).

[4] N. Parekh, V. Ravi Kumar, and B. D. Kulkarni, Chaos 8, 300 (1998); N. Parekh, V. Ravi Kumar, and B. D. Kulkarni, Pramana 48, 303 (1997).

[5] R. Carreto-González, S. Ørstavik, J. Huke, D. S. Broomhead, and J. Stark, Chaos 9, 466 (1999); S. Ørstavik, R. CarretoGonzález and J. Stark, Physica D 147, 204 (2000).

[6] L. Sirovich, Q. Appl. Math. 45, 561 (1987).

[7] P. Holmes, J. L. Lumley and G. Berkooz, Turbulence, Coherent Structures, Dynamical Systems and Symmetry (Cambridge University Press, Cambridge, 1996).

[8] H. Bock, Progress in Scientific Computing, eds. P. Deuflhard and E. Hairer, (Birkhäuser, Boston 1983)2 95; H. G. Bock and K. J. Plitt, A Multiple Shooting Algorithm for Direct Solution of Optimal Control Problems, International federation of automatic control, $9^{\text {th }}$ World congress, Budapest, (1984) (Pergamon, Oxford, 1984).

[9] J. Timmer, Int. J. Bifurcation Chaos. 8, 1505 (1998).

[10] J. Timmer, H. Rust, W. Horbelt, and H.U. Voss, Phys. Lett. A 274, 123 (2000).

[11] E. Baake, M. Baake, H. G. Bock, and K. M. Briggs, Phys. Rev. A 45, 5524 (1992).

[12] M. Bär, R. Hegger, and H. Kantz, Phys. Rev. E 59, 337 (1999).

[13] U. Parlitz and C. Merkwirth, Phys. Rev. Lett. 84, 1890 (2000).

[14] H. Voss, M. J. Bünner and M. Abel, Phys. Rev. E 572820 (1998); H. Voss, in Nonlinear Dynamics and Statistics, Ed. A. Mees, (Birkhaüser, Boston, 2000).

[15] C. López, A. Álvarez and E. Hernández-García, Phys. Rev. Lett. 85, 2300 (2000).

[16] K. Kaneko ed., Theory and Applications of Coupled Map Lattices, (John Wiley \& Sons Ltd., West Sussex, 1993); J. P. Crutchfield and K. Kaneko, Directions in Chaos, (World Scientific, Singapore 1987).

[17] F. H. Willeboordse and K. Kaneko, Physica D 86, 428 (1995).

[18] M. Meixner, S. M. Zoldi, S. Bose and E. Schöll, Phys. Rev. E 61, 1382 (2000).

[19] W. Mazin, K. E. Rasmussen, E. Mosekilde, P. Borckmans, and G. Dewel, Maths. Comp. Simul. 40, 371 (1996).

[20] I. Daubechies, Ten Lectures on Wavelets (SIAM Publications, Philadelphia, 1992).

[21] R. W. Wittenberg and P. Holmes, Chaos 9, 452 (1999).

[22] M.J.D. Powell, A Fast Algorithm for Nonlinearly Constrained Optimization Calculations Lecture notes in Maths, 630, (Springer, Berlin, 1978).

[23] K. Schittkowski, Ann. Operation Res., 5, 485 (1986).

[24] P. E. McSharry and L. A. Smith, Phys. Rev. Lett. 83, 4285 (1999).

[25] A. Hilgers and C. Beck, Europhys. Lett. 45, 552 (1999).

[26] K. Kaneko, Physica D 34, 1 (1989).

[27] S. M. Zoldi and H. S. Greenside, Phys. Rev. Lett. 78, 1687 (1997).

[28] Y. Kuramoto, Chemical Oscillations, Waves and Turbulence, (Springer, Berlin, 1984).

[29] R. J. Field and M. Burger, Oscillations and Travelling Waves in Chemical Systems, (John Wiley, New York, 1985).

[30] P. Gray and S. K. Scott, Chemical Oscillations and Instabilities, (Oxford University Press, 1990).

[31] E. E. Selkov, Eur. J. Biochem. 4, 79 (1968).

[32] K.J. Lee, W.D. McCormick, J.E. Pearson, and H.L. Swinney, Nature 369, 215 (1994).
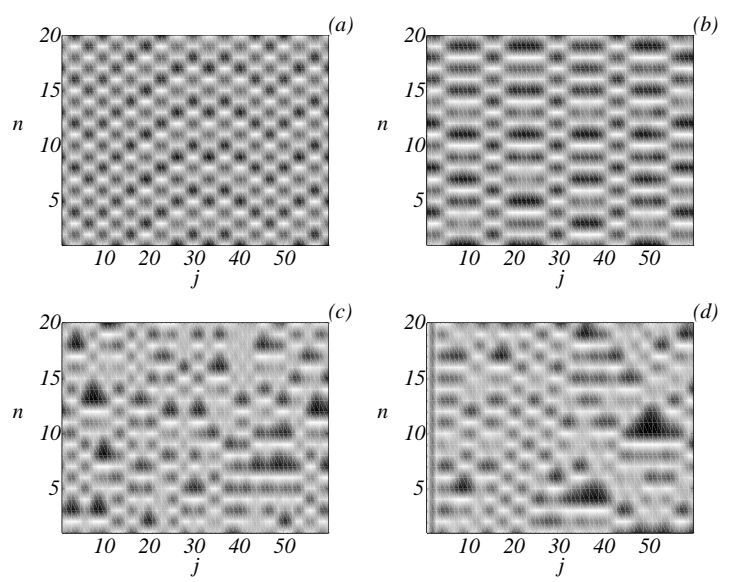

FIG. 1. Evolved spatiotemporal data $u(n, j)$ for the CML ( $j$ spatial grid with $L=60 ; M=20$ snapshots. (a) Weak chaos $\left(F=1.73, D_{d}=0.4, D_{c}=0.0\right)$; (b) Traveling wave $\left(F=1.5, D_{d}=0.5, D_{c}=0.0\right)$; $(c)$ Fully developed chaos $(F=2.0$, $\left.D_{d}=0.4, D_{c}=0.0\right) ;(d)$ Convective turbulence $\left(F=2.0, D_{d}=0.4, D_{c}=0.3\right)$. 

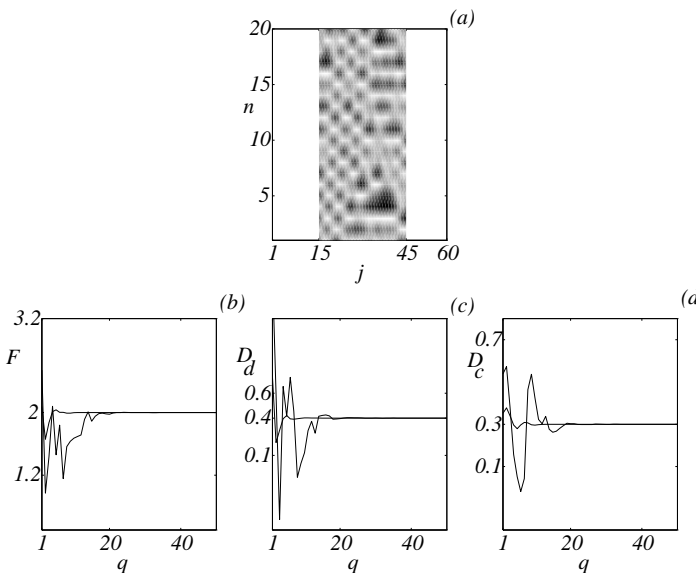

FIG. 2. Parameter estimation for convective turbulence. (a) Subsystem data for the central 31 lattice sites; (b,c,d) Simultaneous convergence to parameter estimates for $F, D_{d}$ and $D_{c}$ for arbitrary initial guesses (shown as $y$-axis labels) as iterations $q$ proceed for minimizing the least square functional.

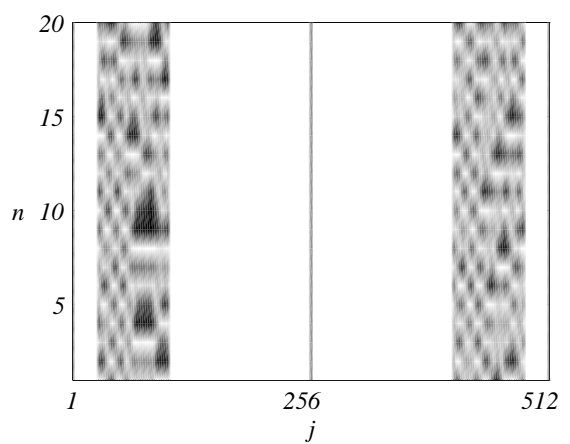

FIG. 3. Data from the left $(F=2.0)$ and the right $(F=1.9)$ subsystems for the inhomogeneous CML. The vertical line at $j=256$ marks the boundary; Other parameter values $D_{d}=0.4, D_{c}=0.3$.

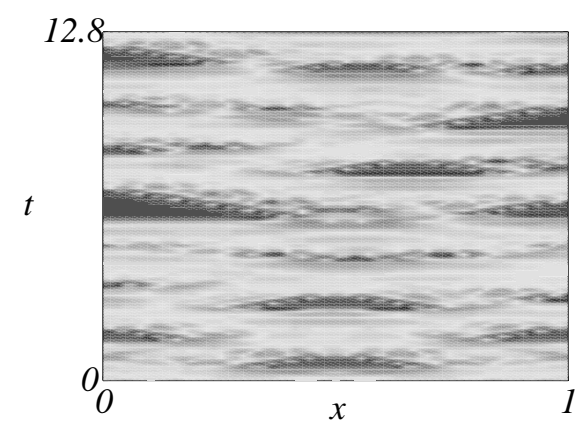

FIG. 4. Spatiotemporal data for the variable $u^{(1)}(t, x)$ in the autocatalytic reaction-diffusion system with parameter values $f=0.029, k=0.0535, D_{u}=0.00002, D_{v}=0.0001$ with spatial length $L=1$ spanning 160 spatial sites and $M=128$ snapshots recorded at a time step $\Delta t=0.1$ is shown. 


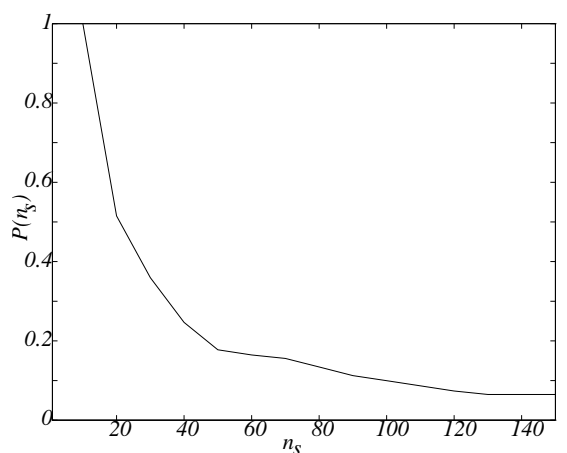

FIG. 5. Power $P\left(n_{s}\right)$ in the first mode of the temporal coefficients, normalized to the maximum, is plotted as a function of subsystem size $n_{s}$.

TABLE I. Significance of KL modes in CML.

\begin{tabular}{llccc}
\hline \hline Case & & Mode no. $k$ & $\lambda_{k}$ \\
\hline (a) & Weakly & 1 & 10.5467 & 0.3639 \\
& chaotic & 2 & 0.2862 & 0.9408 \\
& & 3 & 13.2207 & 0.9988 \\
\hline (b) & Traveling & 1 & 0.3712 \\
& wave & 2 & 0.1098 & 0.9337 \\
& & 5 & 3.8130 & 0.9600 \\
$($ c) & Fully & 1 & 0.0661 & 0.2690 \\
& chaotic & 15 & 0.0217 & 0.9912 \\
& & 19 & 4.0071 & 0.9999 \\
\hline$(d)$ & Convective & 1 & 0.0458 & 0.2950 \\
& turbulence & 15 & 0.0106 \\
\hline \hline
\end{tabular}

TABLE II. Parameter estimation for the CML with varying dynamics. Error bounds for arbitrary initial guesses are shown.

\begin{tabular}{llrr}
\hline \hline Case & & $F$ & $D_{d}$ \\
\hline (a) & Weakly chaotic & $1.73 \pm 0.01$ & $0.40 \pm 0.02$ \\
& with noise & $1.74 \pm 0.03$ & $0.39 \pm 0.02$ \\
(b) & Traveling wave & $1.50 \pm 0.01$ & $0.50 \pm 0.01$ \\
& with noise & $1.54 \pm 0.03$ & $0.53 \pm 0.04$ \\
(c) & Fully chaotic & $1.99 \pm 0.01$ & $0.40 \pm 0.01$ \\
& with noise & $2.03 \pm 0.04$ & $0.38 \pm 0.03$ \\
\hline \hline
\end{tabular}

TABLE III. Parameter estimation from subsystem CML data for convective turbulence. Error bounds for arbitrary initial guesses are shown.

\begin{tabular}{lcccc}
\hline \hline Case & & $F$ & $D_{d}$ & $D_{c}$ \\
\hline (a) & Homogeneous & $1.99 \pm 0.02$ & $0.41 \pm 0.03$ & $0.32 \pm 0.03$ \\
$(b)$ & Inhomogeneous left & $1.99 \pm 0.03$ & $0.39 \pm 0.02$ & $0.29 \pm 0.04$ \\
(c) & Inhomogeneous right & $1.88 \pm 0.03$ & $0.42 \pm 0.04$ & $0.28 \pm 0.04$ \\
\hline \hline
\end{tabular}


TABLE IV. Parameter estimation for the autocatalytic reaction-diffusion system. Error bounds for arbitrary initial guesses are shown.

\begin{tabular}{lcccc}
\hline \hline Case & Data used & Noise level & $f$ & $k$ \\
\hline (a) & $u^{(1)}(x, t)$ & 0.00 & $0.0290 \pm 0.0001$ & $0.0535 \pm 0.0001$ \\
& $u^{(2)}(x, t)$ & 0.02 & $0.0291 \pm 0.0001$ & $0.0537 \pm 0.0001$ \\
& & 0.05 & $0.0296 \pm 0.0003$ & $0.0540 \pm 0.0003$ \\
\hline$(b)$ & $u^{(1)}(x, t)$ & 0.00 & $0.0291 \pm 0.0002$ & $0.0538 \pm 0.0003$ \\
& & 0.02 & $0.0296 \pm 0.0005$ & $0.0565 \pm 0.0003$ \\
\hline$(c)$ & $u^{(1)}(x, t)$ & 0.05 & $0.0303 \pm 0.0008$ & $0.0610 \pm 0.0003$ \\
& $0.25<x<0.75$ & 0.00 & $0.0307 \pm 0.0008$ & $0.0540 \pm 0.0002$ \\
& & 0.02 & $0.0321 \pm 0.0008$ & $0.0578 \pm 0.0002$ \\
\hline \hline
\end{tabular}

